\title{
Improved outcomes in art pregnancies complicated by thrombophilia, treated with low molecular weight heparin in comparison to spontaneously conceived low-risk, untreated pregnancies: a case control study
}

\begin{abstract}
Objective: To compare severe pregnancy complications in ART singleton pregnancies treated with anticoagulation due to thrombophilia to low-risk, spontaneously conceived untreated pregnancies.

Design: Retrospective case control.

Setting: New York Presbyterian-Brooklyn Methodist Hospital, Brooklyn New York.

Patients: 615 ART pregnancies compared with 391 low-risk, untreated pregnancies matched for age and time of delivery.

Interventions: ART pregnancies were treated with LMWH and low dose aspirin daily and managed by MFM specialist during the entire pregnancy.

Main Outcome Measures: Compared for intrauterine growth restriction (IUGR), preterm premature rupture of membranes (pPROM), thrombocytopenia, prematurity, preeclampsia, abruption, and fetal death.

Results: The incidence of IUGR was significantly lower in the ART group $(5.05 \%$ vs. $9.21 \%, \mathrm{p}<0.01)$. Preeclampsia was significantly reduced in the ART group in comparison to the control, $1.14 \%$ vs. $6.39 \%, \mathrm{p}<0.0001$. PPROM was significantly reduced in the ART group in comparison to the control, $1.46 \%$ vs. $7.67 \%, \mathrm{p}<0.0001$. Prematurity was reduced in the ART group, $3.43 \%$ vs. $9.72 \%, \mathrm{p}<0.0001$. Thrombocytopenia incidence was similar, $2.28 \%$ vs. $2.05 \%, \mathrm{p}>0.8$. Abruption was similar in the two groups, ART $1.3 \%$ vs. $1.02 \%$, $\mathrm{p}>0.68$. There were more fetal deaths in the control group but due to the small numbers in both groups, statistical comparison was not possible.

Conclusion: Use of antithrombotics in ART conceived singleton gestations complicated by thrombophilia under MFM specialty care resulted in significantly decreased prevalence of most major obstetrical complications when compared to low-risk unselected untreated pregnancies.
\end{abstract}

Keywords: ART pregnancies, Thrombophilia, In vitro fertilization

\author{
Volume 9 Issue I - 2018
}

\author{
Alexander Kofinas, ${ }^{1,2}$ Brandon Bell,' Jason \\ Kofinas ${ }^{1,3}$ \\ 'New York Presbyterian Brooklyn Methodist Hospital, USA \\ ${ }^{2}$ Kofinas Perinatal PC, USA \\ ${ }^{3}$ Kofinas Fertility Group, USA
}

Correspondence: Alexander Kofinas MD, New York Presbyterian Brooklyn Methodist Hospital, 90I Stewart Avenue, Suite 245, Garden City NY, I I 530, USA, Tel I5 I68320300, Fax 1516832030I,Email ak@kofinas.net

Received: September 23, 2017 | Published: January 05, 2018
Abbreviations: IUGR, Intrauterine Growth Restriction; ART, Assisted Reproductive Technologies; IVF, In Vitro Fertilization; IRB, Institutional Review Board

\section{Introduction}

With the increase of infertility over the last few decades and subsequent increase in the utilization of fertility treatment, there has been an ongoing effort to study the consequences and associations of these interventions on gestation itself as well as the health of the fetus and neonatal outcomes. There have been numerous studies connecting the use of assisted reproductive technologies (ART), the handling of eggs and sperm outside the human body, such as in vitro fertilization (IVF), with adverse pregnancy outcomes. Even after controlling for multiple gestations, increased rates of preeclampsia, gestational diabetes, preterm delivery, and perinatal mortality are noted., ${ }^{1,2}$ Additionally, there have been multiple studies linking ART to negative fetal outcomes such as cerebral palsy, congenital abnormalities, low birthweight, and neurological problems. ${ }^{1,3,4}$ In a recent study, an association between ART pregnancies and autism has been reported. ${ }^{5}$
In the MOSART study ${ }^{6}$ multiple pregnancy outcomes were studied and compared among groups of various degrees of fertility/sub fertility states. This large, longitudinal cohort study concluded ART pregnancy conferred greater risk of gestational hypertension, preterm delivery, low birth weight and SGA infants in a comparison, which controlled for sub fertility as an independent risk factor. Considering our growing reliance on ART treatments, $1.6 \%$ of live births in the US in 2012 are the result of ART use (over 51,000 births and 65,000 infants) ${ }^{7}$ it becomes increasingly important that the reproductive community continues to investigate ways to improve not only ART success in terms of pregnancy and live-birth rate, but also in meaningful improvements in quality perinatal outcomes.

In this study we are looking to compare two groups; one consisting of low-risk spontaneously conceived pregnancies with no antithrombotic treatment and the second consisting of high-risk, ART conceived pregnancies suffering from thrombophilia and being treated with antithrombotics using LMWH and low-dose aspirin under MFM specialty care. While taking into consideration the previously mentioned poor outcomes associated with infertility and 
ART pregnancies in general, it is our goal to study the efficacy of LMWH treatment in decreasing the incidence of adverse perinatal outcomes in pregnancies conceived by means of ART in comparison to a cohort of spontaneously conceived low-risk pregnancies, which were not treated with antithrombotics.

\section{Materials and methods}

We reviewed our perinatal database from January 1st 2010 to December 31st 2015 and extracted all singleton pregnancies, which conceived by means of ART; this included patients conceived by ovulation induction and intrauterine insemination (OI-IUI) and patients conceived by in-vitro fertilization (IVF). We excluded singleton ART gestations not treated with antithrombotics and twin or higher order multiple pregnancies. We identified 710 such pregnancies. Ninety-five patients delivered in other institutions and complete outcomes were available only in 615 who delivered at our own institution. This group formed the case sample. We identified 536 low-risk patients to use as controls. We matched the control patients for age and time of delivery. The control group was selected from the labor and delivery record by selecting the first patient of similar age, plus or minus one year, who delivered a live born or stillborn at 16 weeks gestation or later, following one of our case patients' delivery. We were able to identify only 536 patients because our study group has an average maternal age significantly higher than the general lowrisk population of the hospital. Because of significant age differences between a private perinatal practice and the general hospital population (private and indigent), we were unable to match all study patients with equal number of controls. From the control group we also excluded 57 patients who were treated with antithrombotics for a variety of reasons, and 88 pregnancies that were conceived by ART means. The study design was reviewed by the institutional review board (IRB) and was approved.

The same MFM specialist monitored all ART patients throughout the entire pregnancy. We treated all ART patients with LMWH and low dose aspirin because of existing pro-thrombotic conditions; such pro-thrombotic conditions were identified either as part of the fertility/recurrent pregnancy loss workup prior to conception, or after screening during the first trimester of pregnancy due to ultrasonically identified placental thrombosis ${ }^{8}$ (Table1). The initial LMWH dosage was $40 \mathrm{mg}$ /day and subsequently, it was adjusted according to the maternal serum anti-Xa level. The goal of the treatment was to maintain an anti-Xa level between 0.3 and $0.6 \mathrm{IU} / \mathrm{ml} .{ }^{9}$ Anti-Xa levels were examined every four weeks until 34 weeks gestation. The aspirin was discontinued at 35 weeks gestation and LMWH was replaced by Heparin at 37 weeks gestation and continued until delivery. Platelet counts for both groups were obtained from the delivery admission records for thrombocytopenia comparisons.

Table I Thrombophilic defects identified in the ART patients

\begin{tabular}{ll}
\hline Thrombophilic Defect & Number (\%) \\
\hline Genetic only & $59(9.75)$ \\
Acquired only & $46(7.60)$ \\
Combined (Genetic and acquired) & $500(82.64)$ \\
\hline
\end{tabular}

All collected outcomes data were tabulated accordingly. The prenatal records and hospital charts were reviewed for pertinent information. Demographic data as well as delivery outcomes from the in-hospital records were crosschecked with prenatal records and confirmed for completeness and accuracy. We analyzed the data for prematurity, intrauterine growth restriction (IUGR), preeclampsia, abruptio placentae, fetal death, thrombocytopenia, and pPROM. Comparisons were performed by case vs control.
Preterm birth was defined as any birth $<37$ weeks. IUGR was defined as birth weight at $<10$ th $\%$ for gestation. Preterm PROM was defined as rupture of the amniotic membranes in the absence of labor before 37 weeks gestation. Fetal demise was defined as any spontaneous fetal death after 24 completed weeks of gestation. Preeclampsia was defined according to the criteria of the National High Blood Pressure Education Program Working Group on High Blood Pressure in Pregnancy, adapted by ACOG. ${ }^{10}$

Statistical analysis was done by means of JMP statistical analysis software, SAS Inc., Carry North Carolina. We performed descriptive statistical analysis, simple regression, and one-way analysis of variance for comparison of the means of numerical continuous data. We analyzed categorical data by means of X2 analysis and we set statistical significance at $p$ value of $<0.05$.

\section{Results}

We analyzed 1006 subjects; 391 comprised the control group and 615 the treatment group. The control group consisted of low-risk patients who conceived spontaneously, with singleton pregnancies and who were not treated with antithrombotics. The treatment group consisted of patients who experienced fertility problems with or without recurrent pregnancy loss and conceived the index pregnancy by means of ART. The ART procedures were either IVF or OI-IUI. In the ART group, $54.31 \%$ of the patients conceived by means of IVF and $45.69 \%$ by means of OI-IUI. Among the patients in the ART group, $92.03 \%$ were treated with a combination of LMWH and low-dose aspirin and the rest of the patients with either LMWH alone or aspirin alone. All patients were treated with unfractionated heparin after 37 weeks until the time of delivery. In the ART group, $82.64 \%$ of the patients suffered from combined genetic and acquired thrombophilia, $9.75 \%$ from genetic only, and $7.6 \%$ from acquired only. Mean maternal age was similar in the two groups, $33.1 \pm 0.23$ vs. $33.1 \pm 0.18$ years, $p=0.87$. Gravidity was higher in the ART group than in the control group, $3.1 \pm 0.06$ vs. $2.4 \pm 0.08, \mathrm{p}<0.0001$; parity in contrast was higher in the spontaneously conceived group than the ART group (0.92 vs $0.57, \mathrm{p}<0.001)$.

The incidence of preterm birth, IUGR, preeclampsia, fetal demise, pPROM and abruption were significantly lower in the ART group in comparison to the control group (Table 2).

There were more African-American patients in the control group $(24.55 \%$ vs. $17.24 \%, p<0.001)$. It is known that African Americans suffer higher rates of pregnancy complications than all other ethnic groups in the USA. ${ }^{11}$ Because of the potential effect of this difference in the results of our analysis, we performed a secondary analysis after we excluded all African American subjects. The results of this analysis revealed that the slightly larger proportion of African American patients did not affect the results of the comparison (Table 3).

The incidence of preeclampsia in the ART group was reduced by $82 \%$ in relation to the control group. Likewise, the incidence of pPROM was significantly reduced by $81 \%$ in the ART group. Thrombocytopenia was encountered infrequently in both groups and the incidence was similar, $2.28 \%$ vs. $2.05 \%, \mathrm{p}>0.8$. The level of thrombocytopenia was consistent with pregnancy-induced stable thrombocytopenia and there was no need in the ART group to stop the LMWH in any of the patients. The incidence of abruption in the ART group was similar to the control group, $1.3 \%$ vs. $1.02 \%, p>0.68$. There were more fetal deaths in the control group but due to the small numbers in both groups, statistical comparison was not possible. 
Table 2 Comparison of outcomes between ART and control patients, entire group

\begin{tabular}{lllllll}
\hline Group & PPROM & IUGR & Prematurity & Preeclampsia & Fetal Demise & Abruption \\
\hline ART & $1.46 \%$ & $5.05 \%$ & $3.43 \%$ & $1.14 \%$ & $1.5 / 1000$ & $1.30 \%$ \\
Control & $7.67 \%$ & $9.21 \%$ & $9.72 \%$ & $6.39 \%$ & $5 / 1000$ & $1.02 \%$ \\
P value & $<0.000 \mathrm{I}$ & $<0.01$ & $<0.000 \mathrm{I}$ & $<0.000 \mathrm{I}$ & $\mathrm{ns}$ & $\mathrm{ns}$ \\
\hline
\end{tabular}

Table 3 Comparison of outcomes between ART and control exclusive of African American patients

\begin{tabular}{lllllll}
\hline Group & PPROM & IUGR & Prematurity & Preeclampsia & Fetal Demise & Abruption \\
\hline ART & $1.18 \%$ & $4.33 \%$ & $4.34 \%$ & $0.59 \%$ & $2 / 1000$ & $0.98 \%$ \\
Control & $6.44 \%$ & $9.15 \%$ & $8.14 \%$ & $5.76 \%$ & $3 / 1000$ & $1.36 \%$ \\
P value & $<0.001$ & $<0.008$ & $<0.002$ & $<0.03$ & Ns & ns \\
\hline
\end{tabular}

\section{Discussion}

To our knowledge, this is the first study to compare clinical pregnancy outcomes in ART pregnancies complicated by thrombophilia and treated with low molecular weight heparin and low dose aspirin with outcomes of an unselect group of patients who conceived spontaneously and were never treated with antithrombotics. In this case control study, the control group is not a clinically similar group. However, this does not diminish the value of the findings of this study. To the contrary, it makes the findings more important. It is known that pregnancies conceived by ART have increased rates of IUGR, prematurity as well as other pregnancy complications. ${ }^{1,2,5,12,13}$ Our control group is expected to have lower incidence of complications right in line with national average rates of complications as reported by the $\mathrm{CDC}$ and lower rates of complications in comparison to untreated ART pregnancies.

The lower rate of complications in this control group made it more difficult to demonstrate significant differences in comparison to the ART treated cases. Despite this hurdle, the outcomes of the ART group were so much better that it magnified the significance of the treatment with antithrombotics. The parity and gravidity of the control group was different but in agreement of what was expected, since we did not match the patients for parity and gravidity. The control group exhibited higher rates of parity and lower rates of gravidity; in contrast, the ART group exhibited higher rates of gravidity and lower rates of parity due to the fertility issues and higher rate of pregnancy loss prior to the index pregnancy, a factor that increases the risk for adverse outcomes in subsequent pregnancies even further.

One intervention often offered to patients undergoing ART is the administration of antithrombotic treatment as an adjunctive therapy; it is thought that heparin can potentially modulate many of the known mechanisms that underlie the successful implantation of the developing embryo. Unfractionated heparin as well as LMWH is able to modulate the process of decidualization, potentially improving implantation success in ART conceived pregnancies, as well as, clinical pregnancy rates. ${ }^{14,15}$ Regarding the effects of antithrombotic treatment in pregnancies conceived by ART, the majority of research to date has focused on three primary outcomes: implantation rate, clinical pregnancy rate, and live birth rate..$^{16,17}$ As a consequence, the body of research to date has not addressed the effect of antithrombotic treatment on specific aspects of perinatal outcomes, which are known factors associated with maternal, fetal and neonatal wellbeing.

In our study, we demonstrate significant improvements in the anticoagulant-treated population with regards to various detrimental pregnancy related complications: preterm birth, IUGR, pPROM, and preeclampsia. The incidence of abruption in the ART group was reduced but remained at the same level as the control subjects. Preterm birth, according to the CDC, affects 1 out of every 9 births (11\%) in the US ${ }^{11}$ and is the leading cause of infant death and long-term disabilities such as respiratory difficulties, blindness, developmental delays, cerebral palsy, and impairments in vision and hearing. It is interesting to note that our control group had a preterm delivery rate of $9.72 \%$, less than that of the national average but the ART group experienced even lower prematurity rate at $3.43 \%$.

IUGR in America complicates approximately $8.2 \%$ of all pregnancies; in contrast, the incidence of IUGR in our study group was only $5.05 \%$. IUGR is associated with multiple negative health outcomes during neonatal period and furthermore, in adulthood including hypertension, hyperlipidemia, coronary heart disease, and diabetes. Also, as demonstrated by Bernstein et al. ${ }^{18}$ there is an increased relative risk of neonatal death (RR 2.77), respiratory distress syndrome (RR 1.19), mild intraventricular hemorrhage (RR 1.13) and severe intraventricular hemorrhage (RR 1.27), and necrotizing enterocolitis (RR 1.27). There are many maternal, fetal and environmental factors that contribute to development of IUGR but the common denominator of most frequent causes is variable degrees of placental insufficiency. The relative risk of IUGR, prematurity, preeclampsia, fetal death, and abruption in ART pregnancies can be as high as $3 .{ }^{1}$ In the present study, the magnitude of reduction of the incidence of prematurity, preeclampsia, fetal demise, and IUGR speaks in support of the concept that the cause of the infertility may potentially be the same as the cause of placental insufficiency with all adverse consequences noted in untreated ART pregnancies. ${ }^{19-21}$ LMWH improves implantation rates in patients with thrombophilia; furthermore, LMWH improves pregnancy outcomes and reduces recurrent pregnancy loss in patients with thrombophilic disorders. ${ }^{22,23}$

Preterm premature rupture of membranes (pPROM) occurs in approximately $10-12 \%$ of pregnancies in the United States. ${ }^{24}$ Multiple poor obstetrical outcomes including prematurity, sepsis, pulmonary hypoplasia, umbilical cord prolapse, and placental abruption are the result of pPROM. Risk factors for pPROM among others include decidual ischemia, subchorionic clot formation and placenta pathological states such as, placenta previa, abruptio placentae, and placenta extrachorialis (circumvallate). Such pathologies are amenable to antithrombotic treatments and therefore, their prevention can theoretically reduce the incidence of pPROM. ${ }^{25}$ In our study, we have no other explanation for the reduced incidence of pPROM than the anticoagulant treatment.

Preeclampsia is a common pregnancy-related syndrome with an incidence of $6-10 \%$ in worldwide pregnancies ${ }^{26}$ and a major cause of morbidity and maternal mortality worldwide, with 29,000 maternal deaths in $2013 .^{27}$ There is no clearly understood etiology; however a predominating theory is that abnormally implanted placenta is thought to result in poor uterine and placental perfusion, yielding a state of hypoxia and increased oxidative stress and the release of antiangiogenic proteins into the maternal plasma along with inflammatory 
mediators. ${ }^{28}$ Risk factors for preeclampsia include preexisting hypertension, diabetes, autoimmune diseases such as lupus, various inherited thrombotic diseases, renal disease, women with a family history of pre-eclampsia, obesity, women with a multiple gestation, and women of advanced maternal age. In our study, there was a significant reduction of preeclampsia occurrence to $1.14 \%$ in the study population despite the ART cohort having multiple risk factors, which increase the likelihood of preeclampsia development.

The strength of this study is that it provides an overview of many pregnancy related morbidities in a high-risk cohort and compares them to what should be representative of a general population in America. The fact that the occurrence of many of the major pregnancy related morbidities was decreased can have multiple contributing factors, but the only documented difference is the addition of antithrombotic treatment to their management, under the supervision of a Maternal Fetal Medicine specialist. One weakness however, is that preexisting health conditions and comorbidities were not accounted for with the exception of chronic hypertension, which was similar in the two groups. The control group exhibited the expected incidence of major pregnancy related complications; this makes it very unlikely that the control subjects suffered from more preexisting comorbidities than the ART group.

\section{Conclusion}

This case control study compares an untreated low-risk control group with a high-risk group of pregnancies conceived by ART and in addition, complicated by multifactorial prothrombotic conditions which we treated with LMWH and low dose aspirin. The only substantial difference between these two groups was the use of antithrombotics in the ART group and the fact that a MFM specialist with extensive experience in managing ART conceived pregnancies monitored these patients throughout the entire pregnancy. Therefore, we speculate that the use of LMWH and low dose aspirin under MFM specialty care in ART patients complicated by prothrombotic conditions reduces all major pregnancy related complications to levels that are lower than an untreated low-risk group as well as the national American average as reported by the CDC. Although more data and more studies are needed to confirm our findings, this study strongly reflects potential significant benefits in using antithrombotics in ART pregnancies complicated by prothrombotic conditions in agreement with non-similar but related studies. ${ }^{20,29,30}$

\section{Acknowledgements}

None.

\section{Conflicts of interest}

None.

\section{References}

1. Reddy UM, Wapner RJ, Rebar RW, et al. Infertility, assisted reproductive technology, and adverse pregnancy outcomes: executive summary of a National Institute of Child Health and Human Development workshop. Obstet Gynecol. 2007;109(4):967-977.

2. Schieve LA, Cohen B, Nannini A, et al. A population-based study of maternal and perinatal outcomes associated with assisted reproductive technology in massachusetts. Matern Child Health J. 2007;11(6):517525 .

3. Davies MJ, Moore VM, Willson KJ, et al. Reproductive technologies and the risk of birth defects. N Engl J Med. 2012;366(19):1803-1813.
4. Kovalevsky G, Rinaudo P, Coutifaris C. Do assisted reproductive technologies cause adverse fetal outcomes? Fertil Steril. 2003;79(6):1270-1272.

5. Fountain C, Zhang Y, Kissin DM, et al. Association between assisted reproductive technology conception and autism in california, 19972007. Am J Public Health. 2015;105(5):963-971.

6. Declercq E, Luke B, Belanoff C, et al. Perinatal outcomes associated with assisted reproductive technology: the Massachusetts Outcomes Study of Assisted Reproductive Technologies (MOSART). Fertil Steril. 2015;103(4):888-895.

7. Assisted reproductive technology: Fertility clinic success rates report. CDC, Atlanta, Georgia. 2012.

8. Kofinas A, Kofinas G, Sutija V. The role of second trimester ultrasound in the diagnosis of placental hypoechoic lesions leading to poor pregnancy outcome. J Matern Fetal Neonatal Med. 2007;20(12):859-866.

9. Sarig G, Blumenfeld Z, Leiba R, et al. Modulation of systemic hemostatic parameters by enoxaparin during gestation in women with thrombophilia and pregnancy loss. Thromb Haemost. 2005;94(5):980985.

10. Report of the National High Blood Pressure Education Program Working Group Report on High Blood Pressure in Pregnancy. Am J Obstet Gynecol. 2000;183(1):S1-S22.

11. Joyce AM, Hamilton BE, Ventura SJ, et al. Births: final data for 2009 . National Vital Statistics Reports. 2011;60(1):1-72.

12. Allen VM, Wilson RD, Cheung A. Pregnancy outcomes after assisted reproductive technology. J Obstet Gynaecol Can. 200628(3):220-250.

13. Wang YA, Sullivan EA, Black D, et al. Preterm birth and low birth weight after assisted reproductive technology-related pregnancy in Australia between 1996 and 2000. Fertil Steril. 2005;83(6):1650-1658.

14. Grandone E, Villani M, Dentali F, et al. Low-molecular -weight heparin in pregnancies after ART -a retrospective study. Thromb Res. 2014;134(2):336-339.

15. Qublan H, Amarin Z, Dabbas M, et al. Low-molecular-weight heparin in the treatment of recurrent IVF-ET failure and thrombophilia: a prospective randomized placebo-controlled trial. Hum Fertil (Camb). 2008;11(4):246-253.

16. Akhtar MA, Sur S, Raine-Fenning N, et al. Heparin for assisted reproduction. Cochrane Database Syst Rev. 2013;8:CD009452.

17. Seshadri S, Sunkara SK, Khalaf Y, et al. Effect of heparin on the outcome of IVF treatment: a systematic review and meta-analysis. Reprod Biomed Online. 2012;25(6):572-584

18. Bernstein IM, Horbar JD, Badger GJ, et al. Morbidity and mortality among very-low-birth-weight neonates with intrauterine growth restriction. The Vermont Oxford Network. Am J Obstet Gynecol. 2000;182(1 Pt 1):198-206.

19. Mello G, Parretti E, Fatini C, et al. Low-molecular-weight heparin lowers the recurrence rate of preeclampsia and restores the physiological vascular changes in angiotensin-converting enzyme DD women. Hypertension. 2005;45(1):86-91.

20. Sobel ML, Kingdom J, Drewlo S. Angiogenic response of placental villi to heparin. Obstet Gynecol. 2011;117(6):1375-1383.

21. Lodigiani C, Di Micco P, Ferrazzi P, et al. Low-molecular-weight heparin in women with repeated implantation failure. Womens Health (Lond). 2011;7(4):425-431.

22. Bick RL. Recurrent miscarriage syndrome due to blood coagulation protein/platelet defects: prevalence, treatment and outcome results. DRW Metroplex Recurrent Miscarriage Syndrome Cooperative Group. Clin Appl Thromb Hemost. 2000;6(3):115-125. 
23. Bick RL, Hoppensteadt D. Recurrent miscarriage syndrome and infertility due to blood coagulation protein/platelet defects: a review and update. Clin Appl Thromb Hemost. 2005;11(1):1-13.

24. Beckmann C. Premature rupture of membranes. (6th edn), Obstetrics and Gynecology, Lippincott Williams \& Wilkins, Baltimore, Maryland, USA. 2010; pp.576.

25. Bar J, Mashiah R, Cohen Sacher B, et al. Effect of thrombophylaxis on uterine and fetal circulation in pregnant women with a history of pregnancy complications. Thromb Res. 2001;101(4):235-241.

26. Al Jameil N, Aziz Khan F, Fareed Khan M, et al. A brief overview of preeclampsia. J Clin Med Res. 2014;6(1):1-7.

27. Global, regional, and national age-sex specific all-cause and cause- specific mortality for 240 causes of death, 1990-2013: a systematic analysis for the Global Burden of Disease Study 2013. Lancet. 2015;385(9963):117-171.

28. Steegers EA, Von Dadelszen P, Duvekot JJ, et al. Pre-eclampsia. Lancet. 2010;376(9741):631-644

29. Brenner B, Hoffman R, Blumenfeld Z, et al. Gestational outcome in thrombophilic women with recurrent pregnancy loss treated by enoxaparin. Thromb Haemost. 2000;83(5):693-697.

30. Gris JC, Chauleur C, Molinari N, et al. Addition of enoxaparin to aspirin for the secondary prevention of placental vascular complications in women with severe pre-eclampsia. The pilot randomised controlled NOH-PE trial. Thromb Haemost. 2011;106(6):1053-1061. 